\title{
LA PEDAGOGÍA INTERCULTURAL EN LOS TERRITORIOS INDÍGENAS DE COSTA RICA
}

\author{
Rodrigo Torres Hernández ${ }^{l}$ \\ Julio Morales Campos ${ }^{2}$ \\ Sandra Ovares Barquero ${ }^{3}$
}

"Cuando los indigenas siembran cantan a la naturaleza, al trabajo, al águila o la mariposa. También se danza cuando se prepara la tierra y en el tiempo de

la cosecha".

Juanita Sánchez

\section{Resumen}

Se plantea en este artículo la necesidad de construir en forma conjunta estrategias de pedagogías interculturales, con el fin de revitalizar las lenguas y culturas indígenas de Costa Rica.

Este proyecto se lleva a cabo por parte de la División de Educación Rural del CIDE con docentes itinerantes de lengua y cultura en la comunidad de $\mathrm{Su}$ retka, Talamanca.
Palabras claves: pedagogía intercultural, cosmovisión, maestro de lengua y cultura.

\section{Abstract}

The author comments about the necessity of building intercultural pedagogies jointly, in order to revitalize mother tongues and cultures of Costa Rican indigenous.

This proposal is part of a project carry out by the Education Rural De-

1 Licenciado en I y II ciclos de Educación General Básica con énfasis en Educación Indigena. Actualmente cursa la Maestria en Educación Rural; es docente de ia escuela Suretka, Talamanca.

2 Maestro de lengua bribri en la Escuela Monte Sión, participante del proyecto maestro itinerante de lengua y cultura (Shiroles, Talamanca).

3 Máster en Administración Educativa, especialidad en Curriculum, Enseñanza de las Ciencias y la Biologia. Experiencia en la formación de maestros para comunidades indigenas. Actualmente es académica e investigadora de la División de Educación Rural.

Recibido: 3 de mayo-2007 - Aprobado: 30 de mayo-2007 
partment, which works with culture and mother tongue teachers in the community of Suretka, Talamanca.
Keywords: intercultural pedagogy, cosmo vision, culture and native tongue teacher.

\section{En bribri:}

Ujtè i'ki ikiana che, ì kiana yawè anñita wés sö ìwöblawemirak se' íyiwa, òs se' unjtö enna wés se' serr e' kê chöwa se' ká kivi i' kì.

Kạne i' kaneúk, swöblauk wé kibí patsö, kie DER-CIDE, enna ulítane kós tso' se' kachök e' pa ta.

\section{EI maestro itinerante de lengua y cultura bribri-cabécar}

L

a División de Educación Rural inició hace 15 años, junto con el Departamento de Educación Indígena del Ministerio de Educación Pública, un proyecto de extensión tendiente a conocer las necesidades curriculares en educación formal en las comunidades indígenas de Talamanca y Boruca.

Como producto de este proyecto, nació el Plan de Estudios con énfasis en Educación Indígena, que se llevó a cabo mediante un convenio con el Ministerio de Educación Pública. Hasta la fecha, se han graduado 20 maestros con el grado de Licenciatura; esto permitiría desarrollar de la mano con los indígenas egresados, proyectos de extensión e investigación, por cuanto son conocedores de su lengua y cultura. En la actualidad, se apoya a un grupo de 32 maestros itinerantes de lengua y cultura bribri y cabécar, en el área pedagógica.

Existe una población de docentes nombrados para impartir lengua y cultura, heterogénea por sus características etarias y por su nivel educativo, el cual va desde docentes con primaria incompleta hasta secundaria completa; no se cuenta con ningún maestro que tenga formación universitaria. Por tal razón, los salarios que se pagan responden a la categoría de aspirante, lo que resulta prácticamente un salario simbólico y poca posibilidad de ascenso, así como nombramientos a largo plazo.

En la mayoría de los casos, el conocimiento pedagógico que poseen es el que su experiencia de vida les ha permitido construir, tanto en la cultura como en su lengua; es decir, una cultura e idioma heredados desde la oralidad. 
Un importante porcentaje de maestros indígenas son varones, situación que responde a la necesidad de desplazarse por caminos inseguros y en zonas geográficas de difícil acceso, ya que las lecciones se imparten de manera itinerante en escuelas que se encuentran ubicadas lejos una de la otra; además, se parte del supuesto que para una mujer realizar esta labor, sería más complicada.

La condición laboral de itinerancia obliga a atender varias escuelas en una semana, lo que genera falta de pertinencia e identidad con la comunidad y la escuela. Esto contribuye a no desarrollar arraigo, así como mayores niveles de compromiso; el maestro de lengua y cultura debe interactuar con diferentes docentes en cada escuela.

El trabajo que comparten con sus pares en muchas ocasiones es desvalorizado y no complementado con las temáticas que desarrolla el docente que imparte ciencias, matemática, entre otras. Al no ser asignaturas en las cuales se reporte una nota que signifique la aprobación de un grado escolar, los padres y las madres de familia no les dan la debida importancia, por lo que los docentes deben mantener una incentivación constante para que los niños y las niñas valoren la lengua y la cultura.

En estos procesos, se da un choque metodológico: los niños y las niñas quieren aprender en forma holística y utilizando el entorno escolar de acuerdo con su cultura y visión de mundo. El docente que imparte conocimientos de las asignaturas básicas, lo hace por separado y dentro de cuatro paredes. La pregunta, entonces, es ¿existe relación entre el aprendizaje que desarro a el maestro regular y el docente de lengua y cultura?

\section{Al rescate de nuestra lengua y cultura indígenas}

Aunque en no todos los países, las lenguas indígenas han logrado ser consideradas como oficiales y en algunos otros, como Guatemala, se siga discutiendo esta nueva condición, la legislación de la mayoría de los países de la región reconoce a las poblaciones indígenas el derecho a recibir educación en su lengua ancestral. Así ocurre, por ejemplo, en Argentina, Bolivia, Brasil, Chile, México.

Pese a ello, es muy poco aún lo que se ha avanzado en la realización de este ideal y en el cumplimiento de la legislación. La oficialidad se traduce en el uso parcial de los idiomas indígenas en el espacio educativo formal y en algunos medios de comunicación, fundamentalmente, orales. Se ha hecho muy poco para ir más allá del campo educativo e incorporar los idiomas indígenas 
en otros órdenes de la vida social, para que hombres y mujeres tengan plena participación en la vida política, social y económica de sus países.

Desde 1942, el Instituto Indigenista Interamericano promovió una propuesta educativa para las comunidades indígenas, basada en la integración y la modemización de sus economías, con el fin de facilitar su incorporación al mercado intemacional y superar el rezago económico y social del campesinado indígena (Bolaños, 2002).

Las democracias de América Latina conocen las realidades multiculturales; sin embargo, son escasos los países que enfrentan la realidad de sus sociedades ante un desafio denominado interculturalidad. No hay que limitarse sólo a conocer estas realidades, sino a respetarlas, fortalecerlas y promoverlas como identidades nacionales para facilitar los cambios que orienten a nuevas relaciones, a diálogos, aperturas, a compromisos profundos para descubrir al otro. Es tarea de todos y todas entender y respetar la diversidad cultural y, particularmente, para entender la cultura como la cosmovisión de los pueblos.

Los pueblos indígenas, como una fuerza en algunos países de América Latina y las organizaciones existentes en otros lugares de la región, deben desarrollar una plataforma política de lucha, que lleve a demandas sobre derechos específicos de sus pueblos, sobre aspectos educativos, culturales, ambientales, tierra, salud, economía, desarrollo, espiritualidad, formas de vida, entre otros. Estos reclamos se reflejan en las expresiones más altas en el orden jurídico y en el orden político.

De acuerdo con las investigaciones realizadas, en Costa Rica existen 8 culturas indígenas, distribuidas en 24 territorios legalmente constituidos. Según el censo del año 2000, la población indígena del país es de 63800 personas, de las cuales, un $42 \%$ vive dentro de los territorios indígenas y un $58 \%$, fuera de esos territorios (un $31 \%$ en zonas rurales, generalmente cercanas a los territorios indígenas y un $27 \%$ en el resto del país). Un $21 \%$ de los indígenas vive en zonas urbanas. La población indígena, entonces, constituye un $1,7 \%$ de los casi 4 millones de personas que habitamos este país.

En Costa Rica, oficialmente se reconoce la existencia de 6 lenguas indígenas, aunque en realidad se habla de 8 , porque con el término "guaymi" se encierran dos lenguas (la ngäbére y la burglére) y también hay un importante grupo de misquitos que mantiene su lengua. La huetar, la que parece haber sido la lengua franca a la llegada de los españoles, y la chorotega o mangue, son dos lenguas que se extinguieron en los últimos siglos. 
Las 6 lenguas indígenas, cuya existencia es reconocida, son: la bribri, la cabécar, la guaymí, la malecu o guatuso, la boruca o brunca y la térraba. Las dos últimas están en proceso de extinción y entre las restantes hay diversos niveles de mantenimiento, que varían de una comunidad a otra, e incluso, entre las familias de una misma comunidad.

Por esta razón, los niños ingresan a la escuela con diferentes niveles de conocimiento y uso de su lengua autóctona y del español: un $50 \%$ es monolingüe en la lengua indígena, no habla español o no lo hace de manera funcional; un $40 \%$ es monolingüe en español, es decir, no habla ninguna lengua indígena y un $10 \%$ posee un bilingüismo coordinado, es decir, se comunica fluidamente con igual eficiencia y eficacia en ambas lenguas (Rojas, 2002).

En atención a los diferentes congresos intemacionales de educación intemacional bilingüe, nace en 1990, la iniciativa por parte de la Escuela de Filosofía de la Universidad de Costa Rica de formular un proyecto de enseñanza de la lengua boruca en la Escuela Doris Stone; el éxito y la acogida por parte de esta comunidad le permitieron al Ministerio de Educación (Departamento de Educación Indígena, 1995) incorporar otros territorios con la finalidad de revitalizar y difundir las lenguas indígenas del país, así como rescatar la transmisión oral desde su cosmovisión.

\section{Pueblos indígenas de Costa Rica}

\begin{tabular}{|l|c|c|}
\hline Grupo indigena & Habitantes & Hablantes \\
\hline \hline Cabecares & 9861 & 7622 \\
\hline Bribris & 9636 & 5647 \\
\hline Bruncas o borucas & 2017 & 70 \\
\hline Guaymíes o borucas & 2563 & 2041 \\
\hline Huetares & 1006 & 7 \\
\hline Malecus & 460 & 300 \\
\hline Chorotegas & 868 & 16 \\
\hline Téribes o térrabas & 621 & 57 \\
\hline Total & $\mathbf{2 7 0 3 2}$ & $\mathbf{1 5 7 6 0}$ \\
\hline
\end{tabular}

Fuente: INEC-Instituto Nacional de Estadística y Censos, 2002.

En 1997, mediante la Resolución 34-97, el Consejo Superior de Educación establece como parte del plan de estudios para escuelas indígenas, dos 
asignaturas: lengua indígena, con tres lecciones semanales y cultura indígena, con dos lecciones.

\section{Pedagogía intercultural}

Por consiguiente, construir una pedagogía intercultural es tarea de las instituciones que apoyan estos procesos, lo que apunta a una convivencia y colaboración en la que los diferentes son un valor, cuyo aprovechamiento enriquece la vida de cada uno de los participantes del proceso educativo y de sus propias culturas.

Esta convivencia intercultural, si bien es cierto trasciende los enfoques tradicionales de respeto a las distintas culturas, pasando a la valoración de "lo otro" y al desarrollo de la capacidad de admirarse ante lo distinto y admirar al otro por lo que es, no niega el respeto, sino que lo asume en una dimensión afectiva y holista.

Esta pedagogía apunta a la transformación del currículo oficial para la formación de todos los individuos de la sociedad en las competencias básicas de las diferentes culturas, permitiendo de este modo que cada persona pueda elegir libremente su propio bagaje cultural y ejercer su derecho a participar de todas las culturas, lo que posibilita el intercambio que conduce al progreso de la sociedad.

El respeto por las distintas culturas pasa, necesariamente por el reconocimiento del otro y su circunstancia; pero pasa también -antes y al mismo tiempo-por el reconocimiento de sí mismo y de lo propio. Y para el reconocimiento y valoración de la propia cultura, la identificación de lo distinto es importante. Es este un juego dialéctico en el que la escuela tiene una función dificilmente reemplazable; por eso, es tarea rescatar y aumentar este grupo de hablantes en nuestros territorios.

El proceso de convivencia intercultural en Costa Rica ha obligado a la cultura dominante a reconocer la presencia de culturas distintas en el territorio nacional. Hoy ya no es extraño -como lo era hace sólo 25 años- escuchar hablar de una Costa Rica pluricultural; pero este paso importantísimo es aún insuficiente para el logro de una convivencia intercultural. Se hace necesario dar pasos firmes en las propias identidades culturales de los diversos pueblos, para que desde ahí, sea posible un acercamiento en calidad de iguales a las otras culturas.

En esta perspectiva, se creó la figura del maestro itinerante de lengua y cultura para las escuelas indígenas; en esta misma perspectiva, se inscribe el 
proyecto de capacitación a estos maestros en metodología que den respuesta a su propia realidad cultural. No se tratará de adecuar las estrategias metodológicas de la cultura dominante a la escuela indígena, sino de identificar -y construir- las propias estrategias para la comprensión de su propia cultura.

El proyecto inicia a partir de un diagnóstico participativo desde el cual se construye la propuesta de gestión pedagógica. El trabajo estará centrado en encuentros y talleres, así como en la observación del trabajo cotidiano del maestro itinerante, mediante estrategias de acompañamiento y procesos permanentes de auto y de coevaluación.

El diagnóstico participativo permitió conocer las condiciones generales y de escolaridad en que se encuentran los maestros en esas zonas, así como valorar sus fortalezas, identificar las debilidades y tomar conciencia de los recursos de que disponen en lo personal y en lo colectivo.

En el acercamiento a la población de docentes, será clave la participación de maestros indígenas egresados de la carrera de la División de Educación Rural (DER) del Centro de Investigación y Docencia en Educación de la Universidad Nacional. En primer lugar, porque son sus pares y en educación es sabido que el trabajo entre pares es efectivo por las experiencias curriculares que desarrollan dentro y fuera del entorno escolar. Estas actividades probadas, sistematizadas y comunicadas entre pares, cuentan con un grado elevado de confiabilidad. Además, hay en estos maestros indígenas un conocimiento de su propia cultura que hace posible un diálogo entre iguales, con los maestros de lengua y de cultura.

De la misma forma, será clave en el proceso de acercamiento a los maestros y de desarrollo del proyecto, la participación de la Dirección de Educación Indígena del Ministerio de Educación Pública. En primer lugar, porque es una instancia oficial reconocida por la población con la que se trabajará; en segundo lugar, porque es una instancia que conoce a estas culturas y a estos idiomas; $y$, en tercer lugar, porque la DER ha desarrollado con éxito trabajos con la Dirección de Educación Indígena, en estas comunidades.

Este proceso se atiende a partir de las siguientes necesidades planteadas por los maestros de lengua y cultura como son: recopilación de historias, confección de artesanías, cantos, poesías, así como las estrategias metodológicas pertinentes al aprendizaje de la pedagogía intercultural.

La pregunta que inicia estos encuentros es: ¿Qué funciones cumple como docente de lengua y cultura? Las respuestas se orientan en dar a conocer los diferentes valores autóctonos como: rescate del idioma, confección de las casas tradicionales, elaboración de artesanías, prácticas agrícolas, formas 
de relacionarse con la naturaleza, en otras palabras, la manera colectiva de pensar el mundo, pues existe una lógica distinta en el pensamiento indígena.

En la recopilación de estos conocimientos, el docente pide colaboración a los padres y las madres de familia, así como a los abuelos y otros miembros de las comunidades, con el fin de establecer un puente de diálogo intercultural.

En ocasiones frecuentes, familiarizan estos saberes sentados alrededor del fogón durante días y noches, en las caminatas, en los momentos de siembra, en rituales fúnebres: muchas de estas historias en la actualidad son narradas en las radioemisoras locales o aparecen en los periódicos; algunas de ellas presentan varias versiones, con un hilo conductor común.

La socialización de estos insumos permite dar inicio a la construcción de contextos de aprendizaje interculturales. ¿Cómo incentivar una clase aprovechando este conocimiento de los niños, padres, abuelos y maestros?

En el desarrollo de las diferentes temáticas, el docente ha de explotar la observación, para lo que se realiza una mirada multidimensional: ver lo grande y lo pequeño, lo cercano y lo lejano, los cuatro rumbos del cosmos, es decir, partir de lo concreto.

El aprendizaje se desarrolla por medio de la observación y para realizarla, se emplean los cinco sentidos. Así, la dirección del viento, la ubicación de los nidos en los árboles, el sonido del agua que corre en el río, la siembra de cultivos en forma rotativa, le indican a las comunidades indígenas los beneficios y las medidas que se deben tomar para convivir en armonía con la naturaleza; esta es vista en una forma holística y cíclica. Es de aquí donde se toma el ejemplo para construir sus conocimientos de igual manera. Aprender implica ensayar, por eso, se ensaya en la naturaleza.

En el desarrollo de los talleres, la metodología imperante se ha orientado a:

- Escuchar la palabra de los indígenas, por cuanto han sido condenados al sometimiento y al silencio, sin haber podido expresar su visión de mundo; recoger estas vivencias, socializarlas y sistematizarlas para que sean materia prima de los aprendizajes.

- Ser menos anecdóticos y más profundos en la interpretación de los datos que nos ofrece el pasado o que nos brindan las comunidades indígenas actuales.

- Ser menos descriptivos y más comprensivos en la interpretación de esos datos, renunciando al folclorismo que, con frecuencia, desvía el verdadero sentido de los hechos y disimula la discriminación. 
- Incorporar la concepción del mundo que se expresa con mucha claridad en su teología, por ejemplo Sibú es Gran Creador y Gran Civilizador, además cuando se explica lo espiritual se habla del bien y del mal; el principio del mal está representado por Bi y Ña.

- La educación integral holística y armónica permite un aprendizaje para la vida en convivencia social, así como, una lectura permanente del entorno natural, que le proporciona los elementos para el aprendizaje, como: la utilización de tintes, los cuales utiliza con gran respeto, plantas medicinales, agua, el aire y diversos ecosistemas. La poesía, el canto y la artesanía están cargados de un lenguaje de sabiduría, fundamentado en conceptos filosóficos.

- La creación de ambientes de aprendizaje interculturales en contextos educativos multiculturales y multilingües es un reto para el docente de lengua y cultura.

La pregunta que nos inquieta en el desarrollo de estos talleres es: ¿Qué es el aprendizaje por relación?

\section{A modo de cierre}

- La enseñanza de ambas lenguas debe respetar los intereses del niño y de la niña; deberá ser pertinente y significativa; basarse en metodologías interactivas que lleven al desarrollo lingüistico y cognitivo de ambos.

- $\quad$ El aprendizaje efectivo se da en contextos donde se valora la cultura, la realidad sociolingüística y la personalidad del educando.

- Los materiales didácticos por utilizar deben ser seleccionados con criterios técnicos y pedagógicos. Ellos han de seguir procesos pedagógicos apropiados y han de considerar el desarrollo lingüístico y cognitivo de los niños y las niñas.

- Se recomienda estimular más la producción de textos, teniendo en cuenta que los niños y las niñas desarrollan aún más su lengua a través de la lectura de libros interesantes y relevantes en su propia lengua y que refiejen su contexto cultural.

- A esto deben sumarse los concursos de literatura infantil, con el fin de promover la composición creativa en las lenguas respectivas.

- Se impone la necesidad que en la elaboración de los materiales participen las comunidades, con el fin de que puedan ser considerados y tomados en cuenta sus aportes culturales, económicos y espirituales. 
- La lengua utilizada en la enseñanza debe servir como vehículo para adquirir conocimiento, constituyéndose de este modo en lengua de enseñanza sin dejar de lado su posición.

- Se recomienda la elaboración de distintos diseños de educación bilingüe, de manera que los pueblos o las comunidades puedan elegir el modelo más conveniente, considerando su realidad sociolingüística.

- En lo referente a la normalización de la terminología pedagógica, se recomienda tener en cuenta los criterios lingüísticos históricos, la variación dialectal y, fundamentalmente, el criterio pedagógico mediante el rescate del léxico, el acuñado del léxico dependiendo del campo semántico y el préstamo siguiendo dos caminos: la refonologización o mantenerlo tal como está en la lengua de origen.

- Para que la educación intercultural bilingüe se ajuste a los crecientes cambios socioculturales y lingüísticos, se recomienda especial atención al uso de las perspectivas del género en las lenguas.

- La interculturalidad debe ser un elemento trascendental de toda acción pedagógica. Es decir, lo curricular, lo metodológico, los materiales pedagógicos y hasta la práctica de aula, deberán ser impregnados de este principio que servirá de puente para el respeto y la convivencia práctica de los pueblos.

- También se menciona la necesidad de contar con sistemas de evaluación apropiados al proceso de la enseñanza de las lenguas, debido a que muchas veces los documentos de evaluación no coinciden con los procesos llevados en clase.

- Urge la formación de los docentes responsables de la educación intercultural bilingüe, quienes denotan su poca competencia en la aplicación de metodologías apropiadas para el aprendizaje tanto de la lengua 1 (Ll) como de la lengua 2 (L2).

- En la capacitación de los docentes, se debe incluir el afianzamiento del uso tanto de la L1 como de la L2, porque los docentes, además de las diferencias metodológicas, manifiestan también poca competencia en el uso de las lenguas que se van a enseñar en el aula.

- Resalta la necesidad de brindar espacios de alta capacitación en la temática de la educación intercultural bilingüe, de tal manera que se formen cuadros profesionales especializados que puedan planificar y desarrollar las diversas acciones que requiere la implementación de la educación intercultural bilingüe.

- Las instituciones encargadas de la formación docente deben promover la investigación como metodología de trabajo, ofrecer programas perti- 
nentes y de calidad, para que sus egresados aumenten sus conocimientos acerca de las teorías que sustentan la acción pedagógica.

- La formación de docentes responsables de la educación intercultural bilingüe debe incluir una esmerada atención al componente socioafectivo, así como un análisis exhaustivo de la realidad sociolingüística del país, y los procesos psicolingüísticos por los cuales atraviesan los niños y las niñas bilingües.

- El perfil profesional del docente de la educación intercultural bilingüe contempla que este sea:

- Mediador entre el alumno y el aprendizaje.

- Iniciador e impulsador de actividades significativas.

- Organizador que articula, ordena, coordina el trabajo con sus estudiantes y con la comunidad.

- Investigador que observa, recoge información, reflexiona y evalúa constantemente los procesos de aprendizaje.

- Intercultural, es decir, que sea mediador de las diferentes culturas con las que debe interactuar en las situaciones de aula.

- Democrático, que fomente la participación de sus estudiantes, no sólo en la realización de las actividades conducentes o logros de aprendizaje, sino en la toma de decisiones sobre aspectos que les conciernen y que quieran un ambiente de respeto y que escuchen las opiniones y críticas divergentes.

- Un modelo de formación docente que responda al del profesional reflexivo, intercultural y bilingüe supone ir más allá del modelo meramente cognitivo y tecnológico, implica un compromiso profesional y autocrítica de la práctica docente en el marco de la diversidad. Esta perspectiva concibe un maestro profesional comprometido, que investiga, que analiza y reflexiona sobre su práctica docente.

- Los docentes deben analizar críticamente los problemas de hoy desde una perspectiva amplia y general, para tomar conciencia de los intereses a los que está sirviendo y de aquellos a los que quiere servir.

- El futuro maestro de la educación intercultural bilingüe, como facilitador del aprendizaje, debe ser capaz de adecuar el currículo, planificar, organizar, ejercitar y evaluar situaciones significativas de aprendizaje a partir de las características etnolingüísticas de los niños y las niñas, de su cosmovisión, experiencia y potencialidades. 


\section{REFERENCIAS}

Bolaños, M. (2002). Ruralidad y multiculturalidad en la Centroamérica contemporánea. Ponencia UNA, Costa Rica.

Bozzolli, E. (2000). "La población indigena, la cultura nacional y la cuestión étnica en Costa Rica". Cuadernos de Antropologia. No 8. UCR, San José, Costa Rica.

Rockwell, E. (1998). La dinámica cultural en la escuela. Un enfoque vygostkiano. Madrid, España.

Rodríguez, J. (2002). "Patrones y principios en la moralización de las acciones. Una investigación en la cultura bribri". Revista de Ciencias Sociales. UCR.

Rojas, C. (2002). "La enseñanza de las lenguas indigenas en Costa Rica". Revista Educare $N^{\circ} 111$. Centro de Investigación y Docencia en Educación. UNA.

Segundo, J. (1997). "Relatos Bribris de Kë Köldi". Revista Tradición Oral Indigena Costarricense. Vol. IV. 